\title{
Spatially incongruent sounds affect visual localization in virtual environments
}

\author{
Dong Liu ${ }^{1} \cdot$ Pei-Luen Patrick Rau ${ }^{1}$ \\ Published online: 3 January 2020 \\ (C) The Psychonomic Society, Inc. 2020
}

\begin{abstract}
Distance underestimations along the depth plane are widely found in virtual environments. However, past findings have shown that changes in the visual aspects of virtual reality settings do not lead to more accurate depth estimates. Therefore, we examined if nonvisual stimuli, namely, sounds, could serve as cues that affect observers' depth perception. Accordingly, we conducted two distance discrimination tasks to examine whether observers' depth localization is affected by a spatially incongruent sound. In Experiment 1, a spatially incongruent sound made a visual target appear farther away than a visual target presented with no sound only when a far-distance range (i.e., longer than $12 \mathrm{~m}$ ) was introduced. Experiment 2 further indicated that the sound shifted visual localization only when audiovisual spatial disparity did not exceed $4^{\circ}$. Taken together, our findings suggest that the depth localization of a visual object in virtual reality can be altered by a spatially incongruent sound, and provide a potential approach that we can adopt a spatially incongruent sound as a cue to reduce the depth compression in VR.
\end{abstract}

Keywords Audiovisual spatial incongruence $\cdot$ Distance underestimation $\cdot$ Virtual environments $\cdot$ Visual localization

\section{Introduction}

Vision not only enables one to view color and size but also provides information about depth. Depth perception is crucial for a range of human activities (e.g., estimating the depth distance before throwing a ball to another person), and depth distance can be easily estimated using monocular or binocular cues such as texture gradient and binocular parallax (Howard \& Rogers, 2002). Nevertheless, observers' perceived distances are sometimes quite different from their measured distances. Studies have found that depth perception is largely distorted in virtual environments (Knapp \& Loomis, 2004; Kunz, Wouters, Smith, Thompson, \& Creem-Regehr, 2009; Sahm, Creem-Regehr, Thompson, \& Willemsen, 2005). For example, researchers asked observers to verbally report their estimated depth distances (Knapp \& Loomis, 2004; Kunz et al., 2009) or perform blind walking as depth estimates (Fukusima, Loomis, \& DaSilva, 1997; Li, Phillips, \& Durgin, 2011; Rieser, Ashmead, Talor, \& Youngquist, 1990), and found significant underestimations of measured depth distances in virtual environments.

Pei-Luen Patrick Rau

rpl@mail.tsinghua.edu.cn

1 Department of Industrial Engineering, Tsinghua University, Beijing, China
Researchers have made considerable efforts to explore the possible reasons for distance underestimation in virtual environments. However, previous studies have ruled out the role of some physical or technological factors, such as a limited field of view or graphic rendering quality, because improving the quality of graphic rendering (Kunz et al., 2009; Thompson et al., 2004) or increasing the field of view of a head-mounted display (Knapp \& Loomis, 2004) did not lead to more accurate depth estimates. Considering that changes in the visual aspects of virtual reality (VR) settings were not very effective in correcting observers' depth underestimations, we wonder if nonvisual cues such as sounds can be applied to enhance depth perception accuracy.

Sound is thought to be an effective cue for modulating visual processing in various situations (Shams \& Kim, 2010; Spence, 2011). Nevertheless, most studies on audiovisual spatial interactions, such as the spatial ventriloquism effect, have reported that the perceived location of a sound is usually biased toward a spatially disparate visual stimulus (Bruns \& Röder, 2019; Chen \& Vroomen, 2013). However, it should be noted that audiovisual interactions mostly follow the unity principle, according to which multisensory stimuli that are somewhat discordant in spatial or temporal aspects can be synthesized into a unitary perception (Spence, 2013; Wallace et al., 2004). Based on such a theoretical perspective, sound still has the possibility to act as an influence on visual spatial 
perception (i.e., depth perception) when audio and visual stimuli are spatially incongruent. Indeed, Woods et al. (2011) used a distance estimation task and found that a television (TV) wall was perceived as being closer to the observer when a sound was emitted from a place that was in front of the TV wall, than when no such sound was presented. These findings suggest that a sound with a spatial disparity from the visual target could bias visual localization (i.e., toward the location of the origin of the sound). Therefore, in the present study, we used a spatially discordant sound as a nonvisual cue to test whether depth estimation in virtual environments is affected by spatially discordant sounds.

It has been found that auditory modulation of visual perception is more pronounced in specific locations. For example, auditory information influences visual perception to a greater extent with a far-distance range than with a neardistance range (Van der Stoep, Nijboer, \& Van der Stigchel, 2014). Similarly, a sound that is spatially different from a visual object exerts greater influences on depth localization in the periphery than at the center (Chan, Maguinness, Lisiecka, Setti, \& Newell, 2012). Previous studies have further suggested that the reliability of information from one modality relative to others is not constant at different locations (Alais \& Burr, 2004; Burr \& Alais, 2006; Ernst \& Banks, 2002), which may lead to differences in auditory influences on visual depth perception across different distances. Thus, we further tested how spatially incongruent sounds' influences on visual localization differed with varying depths.

Researchers have found that auditory influences on visual perception are greatly affected by audiovisual spatial disparity. Numerous studies have shown that auditory modulation of visual localization significantly decreases as audiovisual spatial disparity increases (Andre, Corteel, Embrechts, Verly, \& Katz, 2014; Godfroy, Roumes, \& Dauchy, 2003; Komiyama, 1989; Lewald, Ehrenstein, \& Guski, 2001; Wallace et al., 2004). Moreover, such influences may disappear when the audiovisual spatial disparity exceeds a threshold, such as $3^{\circ}$ (Lewald et al., 2001) and $20^{\circ}$ (Komiyama, 1989), in the horizontal plane according to the distance between observers and audiovisual stimuli. Nevertheless, previous research on the influence of audiovisual spatial disparity on visual localization mainly focused on horizontal or vertical planes (van der Stoep, Farnè, Serino, Spence, \& Di Luca, 2016). It is important to understand how audiovisual spatial disparity modulates visual localization along the depth plane.

In the present study, we aimed to examine how sound modulates depth perception in virtual environments by manipulating audiovisual spatial incongruence. Two distance discrimination tasks were conducted in the present study. The objective of the first experiment was to examine how audiovisual spatial incongruence differentially affects visual localization in a near- (less than $5 \mathrm{~m}$ ) or a far-depth (more than 10 $\mathrm{m}$ ) range. Further, as auditory influences on visual localization have been found to be contingent on the degree of audiovisual spatial disparity (Andre, Corteel, Embrechts, Verly, \& Katz, 2014; Godfroy, Roumes, \& Dauchy, 2003; Komiyama, 1989; Lewald, Ehrenstein, \& Guski, 2001; Wallace et al., 2004), we further examined the differences in auditory influences on depth perception across varying disparity levels in Experiment 2.

\section{Experiment 1}

\section{Method}

Participants A total of 48 observers ( 23 women) between the ages of 17 and 31 years (mean age $=23.85$ years, $\mathrm{SD}=3.87$ ) participated in Experiment 1. ${ }^{1}$ One observer was excluded from the study because she could not distinguish between different depths (see the following sections for details). None of the participants declared any hearing impairments and all had normal or corrected-to-normal vision. All participants who participated in this experiment provided written informed consent in accordance with the procedures and protocols mandated by the institutional review board of the University of Tsinghua. All observers were naive to the purpose of the experiments and were compensated at the end of the experiment.

Apparatus and virtual environment We used a head-mounted display from the HTC vive VR System in Experiment 1. The audio was rendered using an audio spatializer SDK plug-in that was built within the unity game engine. A pair of binaural headphones that accompanied the HTC Vive Virtual Reality system was used as the audio display device. Similar to previous studies, audio stimuli presented from different virtual locations via headphones were using non-individualized HRTFs (Finnegan et al., 2016). All sounds that were used in the experiment were pink noise with a sample frequency of $44.10 \mathrm{kHz}$.

In this experiment, we designed a virtual room of $25 \times 5 \times 3$ $\mathrm{m}$ using a unity game engine. The virtual room consisted of three yellow cubes that served as the wall, a gray plane that served as the floor, and a gray-white ceiling. A wood-colored cabinet with the dimensions of $1 \times 1 \times 1 \mathrm{~m}$ was placed on the floor (Fig. 1). The cabinet was placed along a depth plane that had one of the two following distance ranges: $1.50-4.50 \mathrm{~m}$ or $12-8 \mathrm{~m}$. The sound level was set to $60 \mathrm{~dB}$ at the position of the cabinet, $1.50 \mathrm{~m}$ (i.e., near-distance range) or $12 \mathrm{~m}$ (i.e., fardistance range) away from the observer.

\footnotetext{
${ }^{1}$ For all experiments, we sought to collect data from 18-30 participants for a within-subjects design task, as previous studies that used a similar paradigm (Chan et al., 2012; Finnegan, O'Neill, \& Proulx, 2016) have shown ample power for the sample size. In Experiment 1, we conducted a mixed-design task and the sample size for each within-subject group was 24 .
} 


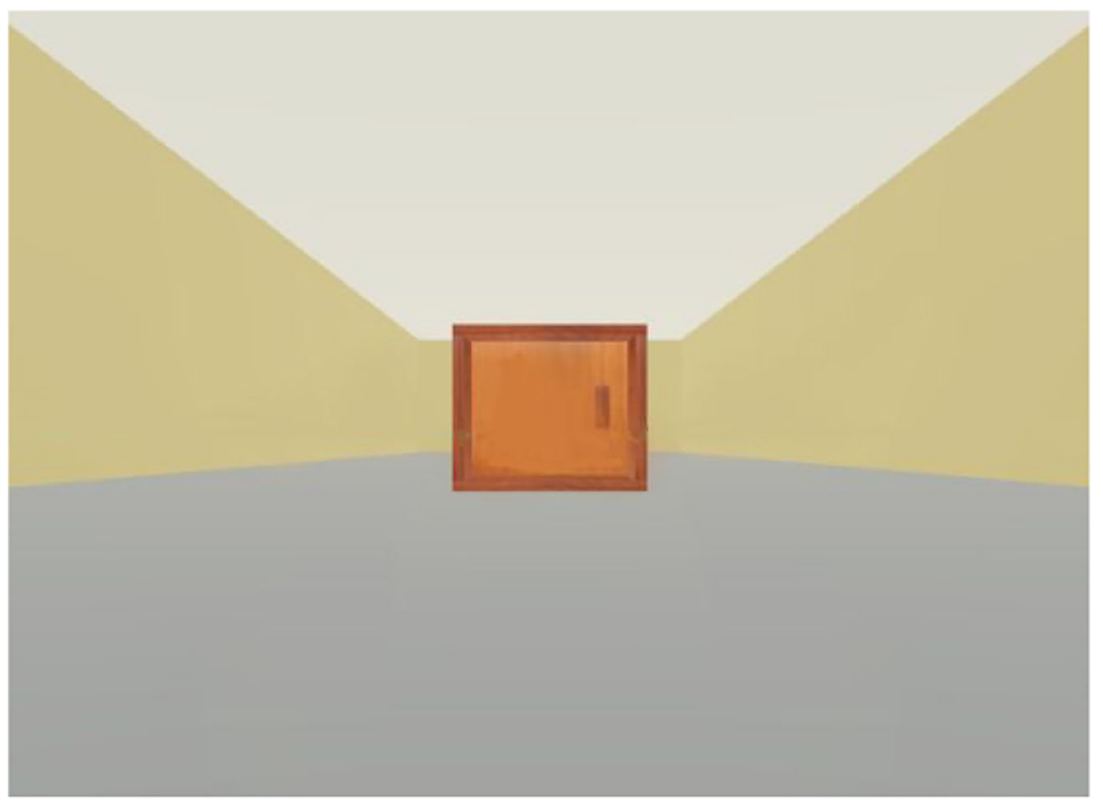

Fig. 1 Image of the virtual environment that was created for Experiment 1. A cabinet was placed along the middle line of the virtual room

Procedure In each trial, one cabinet was presented at a particular distance for $500 \mathrm{~ms}$; after an interval of $300-500 \mathrm{~ms}$, another cabinet appeared for $500 \mathrm{~ms}$. Once the second cabinet appeared, the observers could press a button to indicate which of the two cabinets appeared to be farther away from them. The trial interval was $1,000 \mathrm{~ms}$. Two different distance ranges (i.e., near and far) were used in Experiment 1. One of the cabinets was located at a reference distance ( 3 or $15 \mathrm{~m})$, whereas the other was randomly placed at one of seven test distances: $1.50,2,2.50,3,3.50,4$, and $4.50 \mathrm{~m}$ (reference distance $=3 \mathrm{~m}$ ) or $12,13,14,15,16,17$, and $18 \mathrm{~m}$ (reference distance $=15 \mathrm{~m}$ ); in other words, each distance range entailed seven test conditions. For each trial, one of the two sequentially presented cabinets was randomly chosen to be accompanied by a sound. The sound was presented either at the same location as the cabinet or farther away from the cabinet $(1 \mathrm{~m}$ farther in the near-distance range condition, and $2 \mathrm{~m}$ farther in the far-distance range; hence, the percentages of audiovisual spatial disparities ( 1 or $2 \mathrm{~m}$ ) relative to the largest depth difference among the seven test conditions ( 3 or $6 \mathrm{~m}$ ) were the same in the near- and far-distance ranges). Thus, two comparison sequences (i.e., single-cabinet and cabinet-with-sound) were created during the experiment (see Fig. 2). Before the formal test, each observer had to complete 20 practice trials. Stimuli presentation and the presentation time in the practice were identical to those in the formal test except the positions of the cabinets and sounds.

Experiment 1 adopted a mixed design of distance ranges (near vs. far) and audiovisual spatial incongruence (congruent vs. incongruent). Half of the observers were assigned to the near-distance range condition and the other half to the fardistance range condition. All observers completed two blocks.
In one block, the sound and cabinet were in the same location; in the other, the sound was placed farther away from the cabinet. Each block consisted of 140 trials (i.e., 20 trials per test condition). The order of presentation of the blocks was counterbalanced across the different observers. During the experiment, all observers were required to sit on a chair, and a chin rest was used to keep their heads still.

Data analysis Previous studies asking observers to make an alternative choice in a task had applied the Boltzmann sigmoid function to fit the data (Liu, Yuan, Chen, Jiang, \& Zhou, 2018; Wang \& Jiang, 2012). In the present study, we made conducted a similar analysis to those in previous studies and first calculated the proportions of cabinet-with-sound sequences that were judged as being farther away than the singlecabinet sequence. Furthermore, we fitted the proportion data with the Boltzmann sigmoid function: $\mathrm{f}(\mathrm{x})=1 /[1+\exp (\mathrm{x}-$ $\mathrm{x} 0) / \omega]$; in this equation, $\mathrm{x}$ represents the depth difference between the cabinet-with-sound sequence and the singlecabinet sequence (near-distance range: $-1.50,-1,-0.50,0$, $0.50,1$, or $1.50 \mathrm{~m}$; far-distance range: $-3,-2,-1,0,1,2$, or 3 $\mathrm{m})$, whereas $\mathrm{x} 0$ corresponds to the point of subjective equality (PSE) at which the observer perceived the two sequences as being equal in depth. A negative PSE value in Experiment 1 meant that the observer tends to consider the cabinet-withsound sequence as farther than the single-cabinet sequence, while a positive PSE value indicated the reverse. Meanwhile, half the interquartile range of this fitted function corresponds to the difference limen (DL), which represents the observer's sensitivity to depth discrimination (Fig. 3). Both PSE and DL served as dependent variables and were compared across different conditions and groups. 


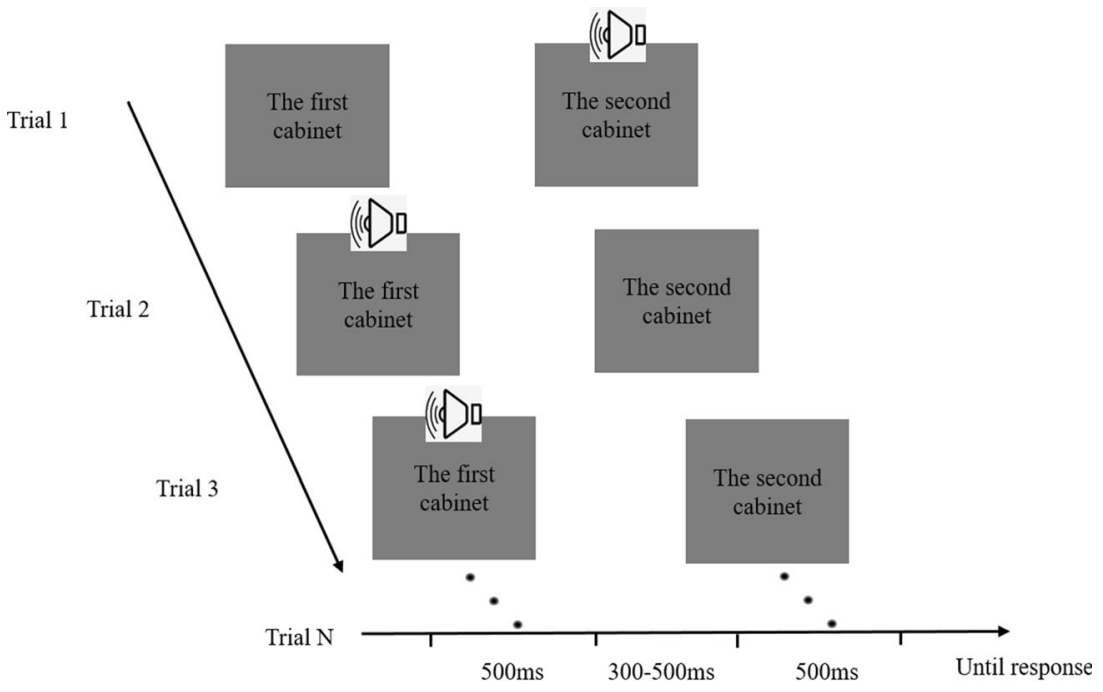

Time course for each trial

Fig. 2 Illustration of the stimuli presentation across different trials. For each trial, one cabinet was randomly chosen to be with a sound and the other was not. Two comparison sequences (the single-cabinet sequence and the cabinet-with-sound sequence) were generated across the whole test

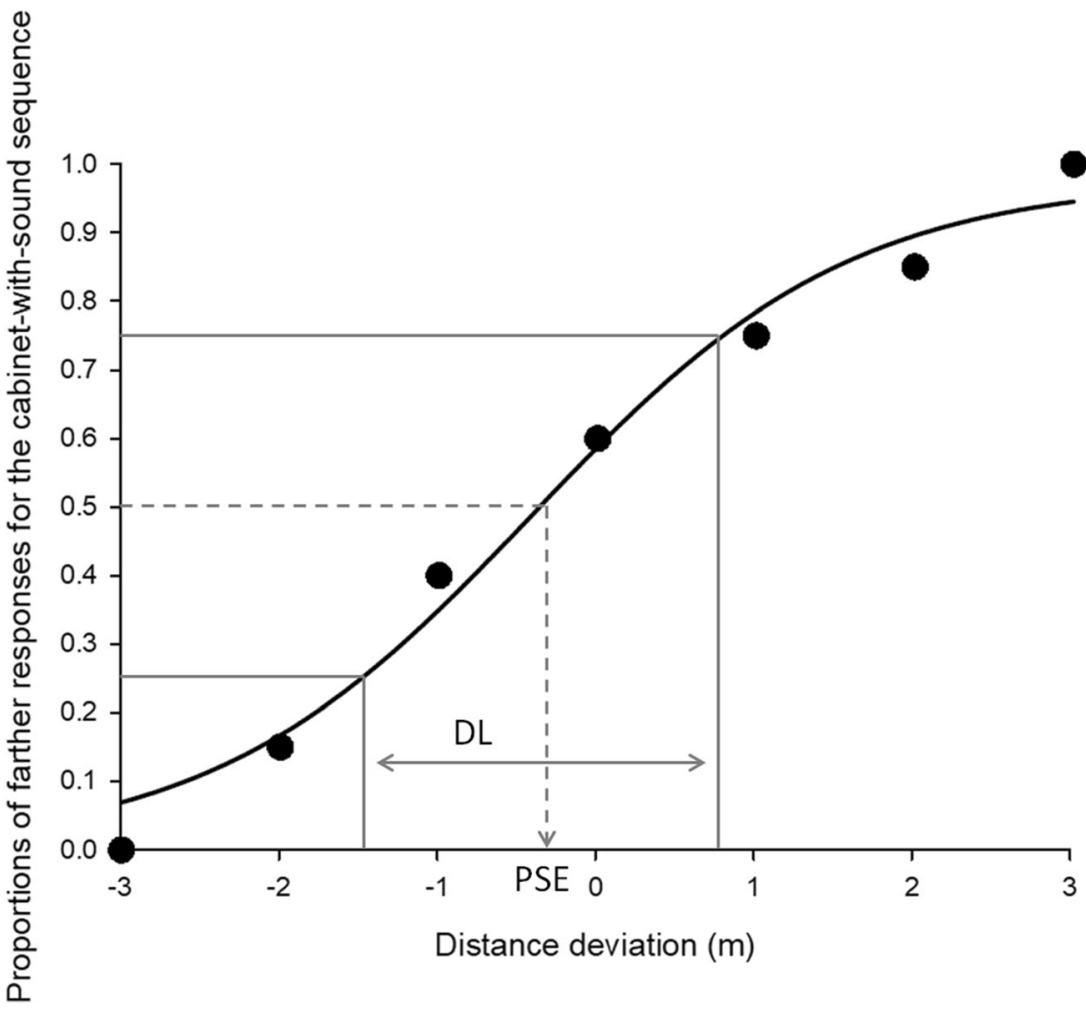

Fig. 3 Illustration of the psychometric function for an observer in an audiovisual spatial incongruent condition with a reference distance of $15 \mathrm{~m}$. The graph shows the proportion of farther responses for the cabinet-with-sound sequence, as a function of the distance differences between the two. The unidirectional arrow represents the point of subjective equality (PSE), whereas the bidirectional arrow represents the difference limen (DL) 


\section{Results}

A 2 (distance ranges: near vs. far) $\times 2$ (audiovisual spatial incongruence: congruent vs. incongruent) repeated-measures analysis of variance was conducted with PSE as the dependent variable. A significant interaction effect was found, $F(1,22)=$ $5.78, p=.025, \eta p 2=.21$. Simple effects analysis further showed that, in the near-distance range condition, PSE was not different between the audiovisual spatial congruent $(\mathrm{M}=$ $3.00, \mathrm{SD}=0.06)$ and incongruent conditions $(\mathrm{M}=3.01, \mathrm{SD}=$ $0.05), \mathrm{t}(23)=-0.62, \mathrm{p}=.539, \mathrm{~d}=0.02$. Meanwhile, in the fardistance range condition, the PSE was significantly lower in the audiovisual spatial incongruent condition $(\mathrm{M}=14.92, \mathrm{SD}=$ $0.12)$ than in the congruent condition $(\mathrm{M}=15.01, \mathrm{SD}=$ $0.14), t(22)=2.39, p=.026, d=0.50$. These findings suggest that audiovisual spatial incongruence affects depth perception when a far-distance range is involved. When the analysis was repeated with DL as the dependent variable, only a main effect of distance range was found to be significant, $F(1,22)=34.81$, $p<.001, \eta_{p}{ }^{2}=.61$. Specifically, observers' sensitivity to depth discrimination was significantly better in the near-distance range than in the far-distance range.

We further tested whether there were significant differences in perceived depth between the single-cabinet sequence and the cabinet-with-sound sequence. We found that the PSE in the near-distance range was not significantly different from 0 in both the audiovisual spatial congruent condition, $t(23)=.26$, $p=.800, d=0.05$, and incongruent condition, $t(23)=1.35, p=$ $.191, d=0.27$. When the cabinet was placed in a far-distance range, the PSE was significantly lower than 0 in the audiovisual spatial incongruent condition, $t(22)=-2.88, p=.009, d=-0.60$, but not in the audiovisual spatial congruent condition, $t(22)=$ $.49, p=.628, d=0.10$ (see Fig. 4). A negative PSE value was found in the audiovisual spatial incongruent condition suggesting that the cabinet-with-sound sequence could be perceived as farther than the single-cabinet sequence. These findings indicate that the cabinet with a spatially discordant sound could affect visual depth perception in a far-distance range.

\section{Experiment 2}

In Experiment 1, we found that an audiovisual spatially incongruent sound alters the perceived location of a visual object; however, the extent to which distance perception is affected by such a sound was not determined. Therefore, in Experiment 2, we directly examined how audiovisual spatial incongruence modulates visual localization by using three different spatial disparity levels. Unlike in Experiment 1, the sound in Experiment 2 was always placed in front of the cabinet for two reasons. One was for the limited distance range of the built virtual room and the other was to eliminate the possibility that sound may alter distance perception in only one direction.

\section{Method}

Participants A total of 30 observers (16 women) between the ages of 17 and 30 years $(\mathrm{M}=23.20$ years, $\mathrm{SD}=3.38)$ participated in Experiment 2. All participants reported having normal or corrected-to-normal vision and normal hearing. All participants in this experiment provided written informed consent, in accordance with procedures and protocols that have been mandated by the institutional review board of the University of Tsinghua.

Procedure and data analysis The apparatus and virtual environments applied in Experiment 2 were same as those in Experiment 1. However, in Experiment 2, only a fardistance range was used; additionally, the sound location was always incongruent with the location of the cabinet, which ranged from 12.60 to $17.40 \mathrm{~m}$. The depth difference between the single-cabinet sequence and the cabinet-withsound sequence was one of the following: $-2.40,-1.60$, $0.80,0,0.80,1.60$, or $2.40 \mathrm{~m}$. Audiovisual spatial disparity was determined by calculating differences between angles that corresponded to the depth distance of the cabinet and those that corresponded to the depth distance of the virtual congruent

incongruent a

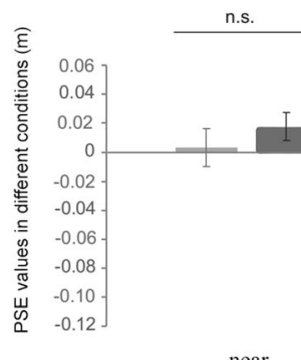

*

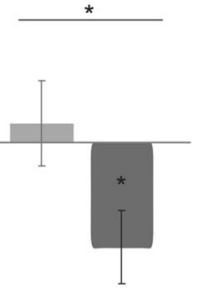

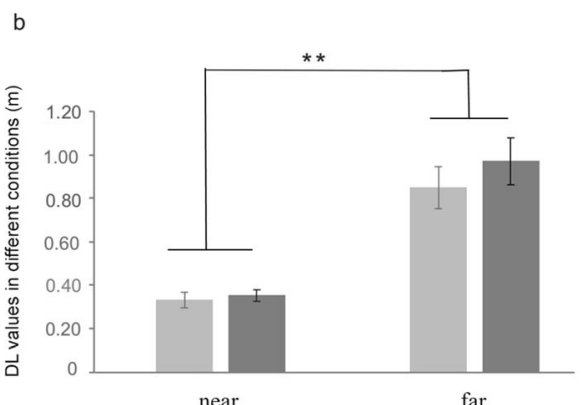

Fig. 4 Depth discrimination results of Experiment 1. Mean point of subjective equality (PSE) (a) and difference limen (DL) (b), as a function of distance range and audiovisual spatial incongruence. n.s. = not significant. $* p<.05 ; * p<.01$. Error bars represent standard errors 
loudspeaker (Fig. 5). Each observer completed three blocks that differed in audiovisual spatial disparity: $2^{\circ}, 4^{\circ}$, and $8^{\circ}$. The order of presentation of the blocks was counterbalanced across participants. In Experiment 2, the proportions of single-cabinet sequence judged as being farther away than the cabinet-with-sound sequence were calculated to generate the PSE and DL values for statistical analyses. A negative PSE value in Experiment 2 meant that the observer tends to consider the single-cabinet sequence as farther than the cabinet-with-sound sequence, while a positive PSE value indicated the reverse.

\section{Results}

We first conducted a one-way repeated-measures ANOVA to directly examine how audiovisual spatial disparity modulates visual distance perception. A significant main effect emerged for PSE, $F(2,58)=5.88, p=.005, \eta_{p}^{2}=.17$. Pairwise comparisons with Bonferroni corrections across three test conditions were conducted, and marginal significant differences were found between the $2^{\circ}(\mathrm{M}=14.89, \mathrm{SD}=0.21)$ and $4^{\circ}(\mathrm{M}=$ $15.01, \mathrm{SD}=0.15$ ) audiovisual spatial disparity conditions, $p=$ .053 , and significant differences were found between the $2^{\circ}$ and $8^{\circ}(\mathrm{M}=15.03, \mathrm{SD}=0.14)$ audiovisual spatial disparity conditions, $p=.013$. However, no significant difference was found between the $4^{\circ}$ and $8^{\circ}$ audiovisual spatial disparity conditions, $p$ $=1.000$. These findings show that the modulating effect of sound on distance perception varies across different audiovisual spatial disparity levels. When the analysis was repeated with $\mathrm{DL}$ as the dependent variable, we did not find a significant main effect for audiovisual spatial disparity, $F(2,58)=.08, p=.927$.
We further tested whether there were significant differences in perceived depth between the single-cabinet and cabinetwith-sound sequences. The results showed that PSE in the $2^{\circ}$ audiovisual spatial disparity condition was significantly smaller than $0, t(29)=-2.93, p=.007, d=-0.53$ (Fig. 6), which implied that the single-cabinet sequence was perceived as being farther away than the cabinet-with-sound sequence, indicating that a sound with a $2^{\circ}$ spatial disparity in front of the cabinet could make the cabinet itself seem nearer than when no sound is presented. In the $4^{\circ}$ and $8^{\circ}$ audiovisual spatial disparity conditions, the PSEs were not significantly different from 0 (disparity $4^{\circ}: t(29)=0.54, p=.594$; disparity $8^{\circ}: t(29)$ $=1.02, p=.317)$. The above findings indicate that there was a spatial limit (possibly less than $4^{\circ}$ ) for the influence of spatially incongruent sounds on visual depth perception.

\section{General discussion}

Sound can influence visual processing in a myriad of ways. Numerous studies have shown that auditory information facilitating visual perception is largely based on matched features (Shams \& Kim, 2010; Spence, 2011, 2013); however, few studies have examined how audiovisual spatial incongruence affects visual perception (e.g., depth perception). In the present study, we adopted two depth discrimination tasks and found that audiovisual spatial incongruence can affect observers' visual localization in virtual environments. Experiment 1 showed that audiovisual spatial incongruence affects depth perception only in a far-distance range (i.e., longer than $12 \mathrm{~m}$ ). Experiment 2 further indicates that audiovisual

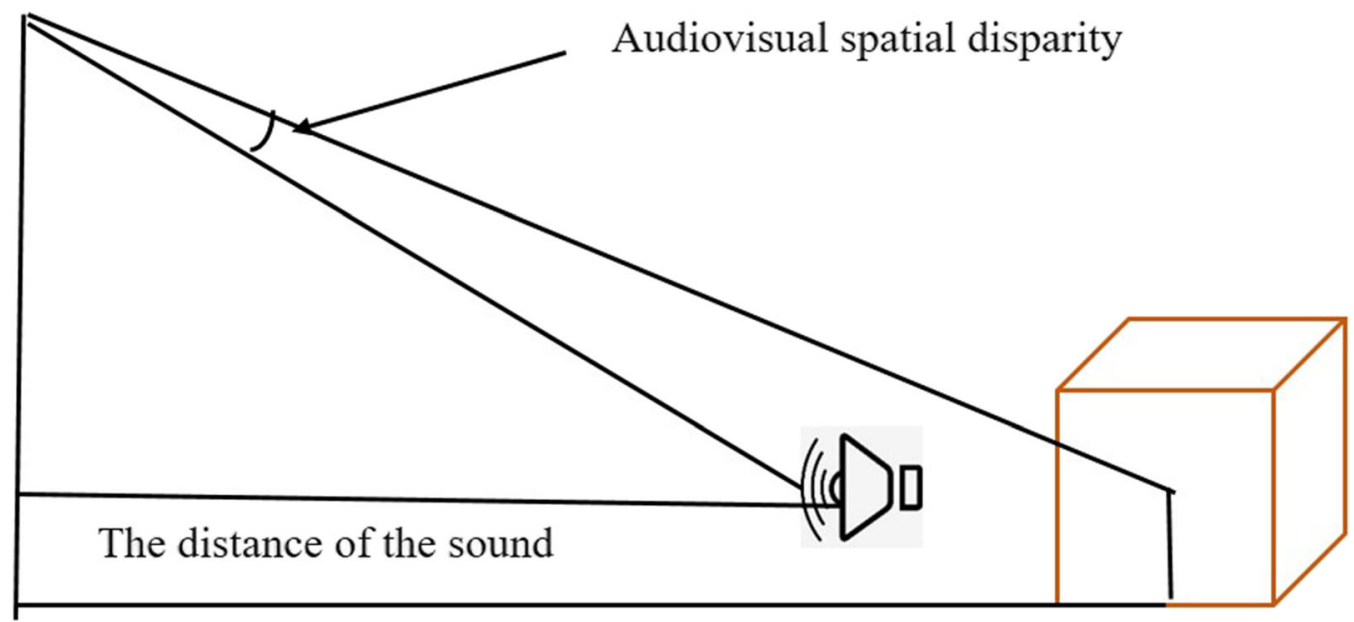

The distance of the cabinet

Fig. 5 Illustration of the calculation of audiovisual spatial disparity in Experiment 2 


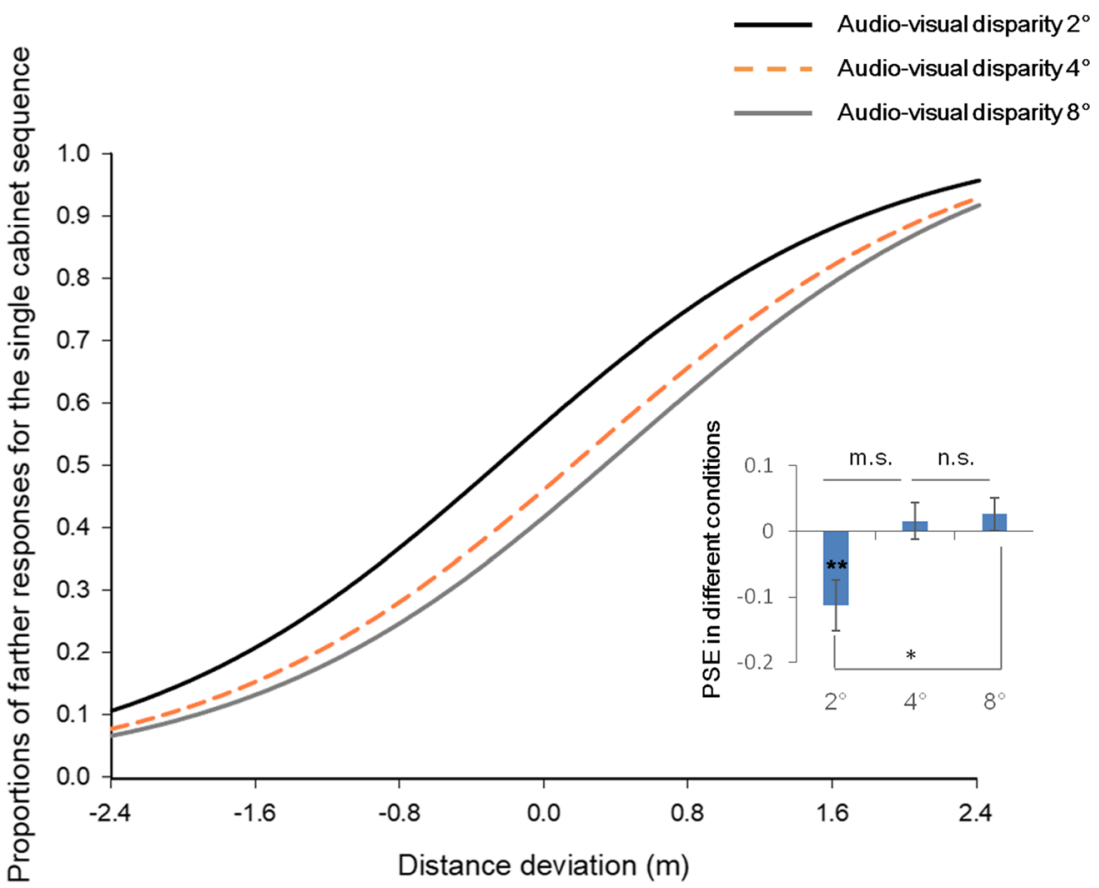

Fig. 6 Depth discrimination results of Experiment 2. Proportion of responses in which observers reported the single-cabinet sequence as farther in depth than the cabinet-with-sound sequence, plotted as a function of the depth difference between the two. Data are shown for the $2^{\circ}$ (gray solid curve), $4^{\circ}$ (orange dashed curve), and $8^{\circ}$ (black dashed curve)

spatial incongruence modulated distance perception only in a limited range (possibly less than $4^{\circ}$ ).

In Experiment 1, audiovisual spatial incongruence affecting depth perception was highly specific to a far-distance range but not a near-distance range. This may be because observers did not rely substantially on audio information when they judged distance differences in a near-distance range. It has been suggested that visual objects with a disparity between $4^{\circ}$ and $7^{\circ}$ can be accurately discriminated in terms of depth (Blakemore, 1970). In the present study, spatial differences between two sequentially presented cabinets in a neardistance range were large enough to allow observers to perform depth discrimination mostly based on visual information. Moreover, the influence of audiovisual spatial incongruence on depth discrimination was found in the far-distance range, suggesting that auditory information can also contribute to visual depth perception.

Previous studies have shown that when audio and visual stimuli are spatially misaligned, the perceived location of the sound was usually shifted to the place of the visual target, even when audiovisual stimuli are presented in the depth dimension (Agganis, Muday, \& Schirillo, 2010; Kitagawa \& Ichihara, 2002). However, in the present study, we found that in the fardistance range, the location of the visual target (e.g., the cabinet) was biased toward the location of the sound. Researchers have found that multisensory perceptual results are largely affected by the reliability of information from each modality relative to others, based on which auditory or haptic information can also audiovisual spatial disparity conditions in Experiment 2. The inset shows the points of subjective equality (PSEs) among the above conditions. Error bars represent standard errors; n.s. $=$ not significant, m.s. $=$ margin ally significant, $* p<.05, * * p<.01$.

have the probability of determining the final perceptual results (Alais \& Burr, 2004; Burr \& Alais, 2006; Ernst \& Banks, 2002). Moreover, this principle for multisensory perception could be applied in both real and virtual environments (Finnegan et al., 2016; Woods et al., 2011; Zhou, Cheok, Qiu, \& Yang, 2007). Hence, it appears that when a far-distance range was introduced, the reliability of auditory information relative to visual information increased, and that auditory information could contribute to the final localization results. Meanwhile, a higher reliability of visual information in the near-distance but not the far-distance range may allow a better depth discrimination performance (i.e., higher sensitivity to depth differences).

Our results confirm Finnegan et al.'s (2016) findings that audiovisual spatial incongruence affects distance perception in VR environments. Specifically, we demonstrated that sound can directly affect distance perception such that the cabinet could be perceived as being nearer or farther away from the observers, based on where the sound was located. Therefore, it is possible to place a sound behind a visual object to make it appear farther away from the observer; this in turn will correct observers' distance underestimations. It should be noted that auditory influences on visual localization were contingent on the degree of audiovisual spatial disparity in the present study. When the spatial disparity exceeded $4^{\circ}$, the sound bias in visual localization disappeared. Thus, the influence range of audiovisual spatial incongruence on visual localization in the depth plane was possibly less than $4^{\circ}$. Nevertheless, the limitation of audiovisual spatial disparity on depth perception (e.g., $4^{\circ}$ ) 
found in the present study occurs in a specific distance range; more research is needed to verify whether such a limitation can be generalized to different scales of distance ranges.

Effective interactions in virtual environments require accurate distance estimations. Researchers have found that a brief period of walking through virtual environments with visual feedback can improve the accuracy of users' distance estimates (Kelly, Cherep, Klesel, Siegel, \& George, 2018; Waller \& Richardson, 2008). Moreover, such improvements can be generalized across different distance ranges (Kelly, Hammel, Siegel, \& Sjolund, 2014) and different virtual environments (Siegel, Kelly, \& Cherep, 2017). Researchers believe that walking through virtual environments can lead observers to rescale their perceived spaces or recalibrate their walking responses so that targets appear farther away after an interaction. However, walking interaction may not be as helpful when virtual environments are constantly changed. Nevertheless, it should be noted that sound can influence visual perception across many situations, even if the visual scenes are dynamically changed (Coutrot \& Guyader, 2013, 2014). Hence, it is possible for us to apply spatially incongruent sounds as cues for enhancing individuals' depth perception accuracy in constantly changing virtual environments (e.g., in the environments of VR games), thus facilitating performance on tasks requiring the judgment of position or distance of objects.

\section{Conclusions}

The present study provided compelling evidence that a spatially incongruent sound can alter visual localization in virtual environments. Specifically, our findings demonstrate that the perceived location of a visual object can be shifted either toward or away from the observer, based on the location of the spatially incongruent sound relative to the visual object. Our findings provide a potential approach that can be used to correct observers' distance underestimates in virtual environments.

Acknowledgements The authors thank Dr. Daniel J. Finnegan for his assistance in writing the experimental program of the present study.

Funding This study was supported by the National Key Research and Development Plan 2016YFB1001200.

\section{Compliance with ethical standards}

Conflict of interest The authors declare no conflicts of interest.

\section{References}

Agganis, B. T., Muday, J. A., \& Schirillo, J. A. (2010). Visual biasing of auditory localization in azimuth and depth. Percept Mot Skills, 111(3), 872-892. https://doi.org/10.2466/22.24.27.PMS.111.6.872892
Alais, D., \& Burr, D. (2004). The ventriloquist effect results from nearoptimal bimodal integration. Current Biology, 14(3), 257-262. https://doi.org/10.1016/j.cub.2004.01.029

Andre, C. R., Corteel, E., Embrechts, J.-J., Verly, J. G., \& Katz, B. F. G. (2014). Subjective evaluation of the audiovisual spatial congruence in the case of stereoscopic-3D video and Wave Field Synthesis. International Journal of Human-Computer Studies, 72(1), 23-32. https://doi.org/10.1016/j.ijhcs.2013.09.004

Blakemore, C. (1970). The range and scope of binocular depth discrimination in man. J Physiol, 211(3), 599-622.

Bruns, P., \& Röder, B. (2019). Cross-Modal Learning in the Auditory System. In A. K. C. Lee, M. T. Wallace, A. B. Coffin, A. N. Popper, \& R. R. Fay (Eds.), Multisensory Processes: The Auditory Perspective (pp. 221-242). Cham: Springer International Publishing.

Burr, D., \& Alais, D. (2006). Combining visual and auditory information. Prog Brain Res, 155, 243-258. https://doi.org/10.1016/S00796123(06)55014-9

Chan, J. S., Maguinness, C., Lisiecka, D., Setti, A., \& Newell, F. N. (2012). Evidence for crossmodal interactions across depth on target localisation performance in a spatial array. Perception, 41(7), 757773. https://doi.org/10.1068/p7230

Chen, L., Vroomen, J., (2013) Intersensory binding across space and time: A tutorial review. Attention, Perception, \& Psychophysics 75(5), 790-811

Coutrot, A., \& Guyader, N. (2013). Toward the Introduction of Auditory Information in Dynamic Visual Attention Models. 2013 14th International Workshop on Image Analysis for Multimedia Interactive Services (Wiamis).

Coutrot, A., \& Guyader, N. (2014). How saliency, faces, and sound influence gaze in dynamic social scenes. Journal of Vision, 14(8). https://doi.org/10.1167/14.8.5

Ernst, M. O., \& Banks, M. S. (2002). Humans integrate visual and haptic information in a statistically optimal fashion. Nature, 415(6870), 429-433. https://doi.org/10.1038/415429a

Finnegan, D. J., O'Neill, E., \& Proulx, M. J. (2016). Compensating for Distance Compression in Audiovisual Virtual Environments Using Incongruence. 34th Annual Chi Conference on Human Factors in Computing Systems, Chi 2016, 200-212. https://doi.org/10.1145/ 2858036.2858065

Fukusima, S. S., Loomis, J. M., \& DaSilva, J. A. (1997). Visual perception of egocentric distance as assessed by triangulation. Journal of Experimental Psychology-Human Perception and Performance, 23(1), 86-100. https://doi.org/10.1037/0096-1523.23.1.86

Godfroy, M., Roumes, C., \& Dauchy, P. (2003). Spatial variations of visual-auditory fusion areas. Perception, 32(10), 1233-1245. https://doi.org/10.1068/p3344

Howard, I. P., \& Rogers, B. J. (2002). Seeing in depth, Vol. 2: Depth perception. Toronto, ON, Canada: University of Toronto Press.

Kelly, J. W., Cherep, L. A., Klesel, B., Siegel, Z. D., \& George, S. (2018). Comparison of Two Methods for Improving Distance Perception in Virtual Reality. ACM Transactions on Applied Perception, 15(2). https://doi.org/10.1145/3165285

Kelly, J. W., Hammel, W. W., Siegel, Z. D., \& Sjolund, L. A. (2014). Recalibration of perceived distance in virtual environments occurs rapidly and transfers asymmetrically across scale. IEEE Trans Vis Comput Graph, 20(4), 588-595. https://doi.org/10.1109/TVCG. 2014.36

Kitagawa, N., \& Ichihara, S. (2002). Hearing visual motion in depth. Nature, 416(6877), 172-174. https://doi.org/10.1038/416172a

Knapp, J. M., \& Loomis, J. M. (2004). Limited field of view of headmounted displays is not the cause of distance underestimation in virtual environments. Presence: Teleoper. Virtual Environ., 13(5), 572-577. https://doi.org/10.1162/1054746042545238 
Komiyama, S. (1989). Subjective Evaluation of Angular Displacement between Picture and Sound Directions for Hdtv Sound Systems. Journal of the Audio Engineering Society, 37(4), 210-214.

Kunz, B. R., Wouters, L., Smith, D., Thompson, W. B., \& Creem-Regehr, S. H. (2009). Revisiting the effect of quality of graphics on distance judgments in virtual environments: a comparison of verbal reports and blind walking. Atten Percept Psychophys, 71(6), 1284-1293. https://doi.org/10.3758/APP.71.6.1284

Lewald, J., Ehrenstein, W. H., \& Guski, R. (2001). Spatio-temporal constraints for auditory-visual integration. Behavioural Brain Research, 121(1-2), 69-79. https://doi.org/10.1016/S0166-4328(00)00386-7

Li, Z., Phillips, J., \& Durgin, F. H. (2011). The underestimation of egocentric distance: evidence from frontal matching tasks. Atten Percept Psychophys, 73(7), 2205-2217. https://doi.org/10.3758/s13414011-0170-2

Liu, R., Yuan, X., Chen, K., Jiang, Y., \& Zhou, W. (2018). Perception of social interaction compresses subjective duration in an oxytocindependent manner. Elife, 7. https://doi.org/10.7554/eLife.32100

Rieser, J. J., Ashmead, D. H., Talor, C. R., \& Youngquist, G. A. (1990). Visual perception and the guidance of locomotion without vision to previously seen targets. Perception, 19(5), 675-689. https://doi.org/ 10.1068/p190675

Sahm, C. S., Creem-Regehr, S. H., Thompson, W. B., \& Willemsen, P. (2005). Throwing versus walking as indicators of distance perception in similar real and virtual environments. ACM Trans. Appl. Percept., 2(1), 35-45. https://doi.org/10.1145/1048687.1048690

Shams, L., \& Kim, R. (2010). Crossmodal influences on visual perception. Phys Life Rev, 7(3), 269-284. https://doi.org/10.1016/j.plrev. 2010.04.006

Siegel, Z. D., Kelly, J. W., \& Cherep, L. A. (2017). Rescaling of perceived space transfers across virtual environments. J Exp Psychol Hum Percept Perform, 43(10), 1805-1814. https://doi.org/10.1037/ xhp0000401

Spence, C. (2011). Crossmodal correspondences: a tutorial review. Atten Percept Psychophys, 73(4), 971-995. https://doi.org/10.3758/ s13414-010-0073-7

Spence, C. (2013). Just how important is spatial coincidence to multisensory integration? Evaluating the spatial rule. Ann N Y Acad Sci, 1296, 31-49. https://doi.org/10.1111/nyas.12121
Thompson, W. B., Willemsen, P., Gooch, A. A., Creem-Regehr, S. H., Loomis, J. M., \& Beall, A. C. (2004). Does the quality of the computer graphics matter when judging distances in visually immersive environments. Presence: Teleoper. Virtual Environ., 13(5), 560 571. https://doi.org/10.1162/1054746042545292

van der Stoep, N., Farnè, A., Serino, A., Spence, C., \& Di Luca, M. (2016). Depth: the Forgotten Dimension in Multisensory Research. Multisensory Research, 29(6-7), 493-524. https://doi.org/10.1163/ 22134808-00002525

Van der Stoep, N., Nijboer, T. C., \& Van der Stigchel, S. (2014). Exogenous orienting of crossmodal attention in 3-D space: support for a depth-aware crossmodal attentional system. Psychon Bull Rev, 21(3), 708-714. https://doi.org/10.3758/s13423-013-0532-y

Wallace, M. T., Roberson, G. E., Hairston, W. D., Stein, B. E., Vaughan, J. W., \& Schirillo, J. A. (2004). Unifying multisensory signals across time and space. Exp Brain Res, 158(2), 252-258. https://doi.org/10. 1007/s00221-004-1899-9

Waller, D., \& Richardson, A. R. (2008). Correcting distance estimates by interacting with immersive virtual environments: effects of task and available sensory information. $J$ Exp Psychol Appl, 14(1), 61-72. https://doi.org/10.1037/1076-898X.14.1.61

Wang, L., \& Jiang, Y. (2012). Life motion signals lengthen perceived temporal duration. Proc Natl Acad Sci U S A, 109(11), E673-677. https://doi.org/10.1073/pnas.1115515109

Woods, A. J., Turner, A., Holliman, N. S., Berry, J., Holliman, N., \& Dodgson, N. A. (2011). Can the perception of depth in stereoscopic images be influenced by 3D sound? Displays, 7863. https://doi.org/ $10.1117 / 12.871960$

Zhou, Z., Cheok, A. D., Qiu, Y., \& Yang, X. (2007). The Role of 3-D Sound in Human Reaction and Performance in Augmented Reality Environments. IEEE Transactions on Systems, Man, and Cybernetics - Part A: Systems and Humans, 37(2), 262-272. https://doi.org/10.1109/tsmca.2006.886376

Publisher's note Springer Nature remains neutral with regard to jurisdictional claims in published maps and institutional affiliations. 